\title{
Evaluation of Bone Capital After Orthodontic- Surgical Traction of the Maxillary Impacted Canines: A Systematic Review
}

\author{
Abdoul Hafizou Rabe (iD, Abdelali Halimi, Fatima Salek, Mohamed Faouzi Azaroual \\ Asmae Benkaddour, Fatima Zaoui \\ Department of Dentofacial Orthopedics, Ibn-Sina Hospital Center, Faculty of Dental Medicine \\ Mohammed V University, Rabat, Morocco.
}

\begin{abstract}
Background: The aim of our study is to evaluate, through a systematic review, the bone capital of the maxillary impacted canines vestibular or palatine trailed compared to their contralateral counterpart. Method: five databases were consulted: PubMed; PubMed Central, Google Scholar, Cochrane Library, and Ebscohost. The research included published studies from 2010 to 2018, meta-analysis studies, randomized and non-randomized controlled trials, prospective and retrospective studies, case series, control cases. Results: Among 299 selected references, only 4 studies met our inclusion criteria. There is no statistically significant difference in alveolar bone loss between the two closed and open techniques for palatal canine traction. Bone loss ranged from $0.03 \mathrm{~mm}$ to $0.39 \mathrm{~mm}$ compared to the contralateral normal canine. For vestibular traction, bone resorption compared to the normally located canine varies from 0.82 to $0.89 \mathrm{~mm}$. Conclusion: Canine traction is accompanied by a statistically significant bone loss compared to the contralateral canine. This bone defect is more aggravated when the inclusion is vestibular, but remains clinically acceptable
\end{abstract}

KEYWORDS: Bone Capital; Maxillary Impacted Canine; Orthodontic Traction.

Correspondence: Dr Abdoul Hafizou Rabe, Department of Dentofacial Orthopedics, Ibn-Sina Hospital Center, Faculty of Dental Medicine, Mohammed V University, Rabat, Morocco. Email : abdoulrabeamani@gmail.com

Copyright (C) 2020 Rabe AH et al. This is an open access article distributed under the Creative Commons Attribution 4.0 International, which permits unrestricted use, distribution, and reproduction in any medium, provided the original work is properly cited.

\section{INTRODUCTION}

The maxillary canines are the most commonly impacted teeth, just behind the third molars [1]. Inclusion of the maxillary canine occurs in about $2 \%$ of the general population, it is twice as common in women as in men and twice as common in the maxilla than in the mandible. For maxillary inclusion, one-third of included canines have a vestibular localization and two-thirds have a palatal localization [2]. Several etiological factors are involved in canine inclusion: local, systemic, or genetic factors, the difference between the size of the canine inclusions and the space required on the arch. Indeed, Jacoby found that only $17 \%$ of maxillary canines with vestibular inclusion had sufficient space, while $85 \%$ of canines with palatal inclusion had sufficient space on the arch [1,2]. Three surgical techniques are frequently used to pull a vestibular included canine tooth: gingivectomy, apically displaced flap, and closed technique [3]. And two surgical techniques are used for palatal traction: open and closed technique [4].

One of the fundamental indicators of the traction success of an impacted tooth is its periodontal status in general, and its bone capital in particular after post-treatment alignment with its contralateral counterpart. A recent randomized controlled clinical trial conducted by Parkin et al [5] compared the different surgical procedures for canine palatal traction. And a systematic review conducted by S. Incerti-Parenti et al [6] evaluated periodontal health after vestibular traction. To date, no review has been carried out to evaluate the bone capital after canine traction of both vestibular and palatal inclusions to our knowledge. The aim of our study is to evaluate through a systematic review of the bone capital of the vestibular or palatal included canine maxillary inclusions pulled compared to their contralateral counterpart.

\section{MATERIALS AND METHODS}

The search for both inclusion and exclusion criteria was based on the PICOT format (Table 1). Electronic searching of the articles in this systematic review was conducted through the PubMed Central, Cochrane Library, Google Scholar, and Ebscohost databases. The PICOT format (Table 1) was used to develop the search strategy. Articles 
published since 2010 were analyzed. No limitations on the language of publication were imposed.

Boolean operators "OR" and "AND" were used to define and connect search terms.

Table 1. PICOS criteria

\begin{tabular}{lll}
\hline Component & Description \\
\hline Population & $\begin{array}{l}\text { Patients requiring orthodontic } \\
\text { traction }\end{array}$ & $\begin{array}{l}\text { Unilateral maxillary canine included } \\
\text { traction }\end{array}$ \\
Comparaison & $\begin{array}{l}\text { Controlateral canine } \\
\text { bone capital } \\
\text { Outcome }\end{array}$ & $\begin{array}{l}\text { No clinical case reports, } \\
\text { interventional or observational }\end{array}$ \\
& $\begin{array}{l}\text { human studies with specific data on } \\
\text { bone capital of the unilateral } \\
\text { maxillary canine included after } \\
\text { orthodontic traction }\end{array}$ \\
\hline
\end{tabular}

\section{Selection of Studies}

Systematic searches were carried out by an author (AHR). The selection of studies was done by two authors (AHR and $\mathrm{AH}$ ). Titles and abstracts were read and the studies were then evaluated against the eligibility criteria. The two authors independently evaluated the selected studies for eligibility. Papers that met the criteria were selected to be read in full text. In case of disagreement between authors, the study was selected for full-text reading. We included studies meeting all of the following criteria: studies published since 2010, meta-analysis studies, randomized and non-randomized controlled trials, prospective and retrospective studies, studies concerning orthosurgical treatment of unilateral canine inclusion.* We excluded all publications on animals, literature reviews, narrative reviews, opinion articles, studies on patients with syndromic or severe facial deformities, studies on patients treated in the mixed or temporary denture.

\section{Database and search strategy}

Electronic searching of the articles in this systematic review was conducted through PubMed, PubMed Central, Cochrane Library, Google Scholar, and EBSCOhost databases. We used the keywords present in the MeSH for the English-language articles, according to the search equation ((impacted canines) AND (Periodontal Status* OR periodontal response* OR periodontal effect*) AND (orthodontics OR method* or Surgical-Orthodontic Treatment )). and for the articles in French, we used the following equation ((canines included) AND (periodontal status or periodontal response or periodontal effect) AND (orthodontics or orthosurgical treatment or traction)). To extract data from the selected articles, we used a table to be reported for each study: type of study, sample size, type of intervention and means of bone assessment, results (table 2). In case of disagreement; the article was discussed with the other authors.

\section{RESULTS}

The search with keywords gave the following results: PubMed produced 8 publications; PubMed Central 32 publications, Google scholar 244, Cochrane Library 4, and Ebscohost 11 publications.

After excluding 13 repeat articles, all titles and abstracts were read and those found to be unrelated to the journal were eliminated, six pre-selected articles were read in their entirety, and after applying the inclusion and exclusion criteria, five references were selected for this systematic review.

The selection process is illustrated in the Flowchart (Fig 1).

Of the four selected studies included in the systematic review:

-one study was prospective controlled [7]

-one study was prospective [8].

-two studies were retrospective $[9,10]$.

The four eligible studies included a total of 133 patients who underwent a surgical uncovering of the maxillary impacted canine. Approximately $63 \%$ of the patients were female with a mean age range of 14 years. Three out of four studies evaluated the bone outcome of palatine inclusive canine traction [7-9]. The prospective controlled study by D. Smailiene et al [7] evaluated the periodontal status between open and closed techniques in 43 patients using two surgical techniques: open and closed techniques (Table 2). Groups 1 (OTE) and 2 (CTE) were homogeneous with respect to the initial vertical and horizontal positions of impacted canines on the panoramic radiographs and the patient's age at the start of treatment. The angle of inclination of the impacted tooth was $38.5 \pm 14.02$ degrees in group 1 and $34.33 \pm 14.57$ degrees in group 2. With respect to the vertical position of the impacted teeth, 81.8 percent (18) in group 1 and 61.9 percent (13) in group 2 were situated in sector V1. The mean time required to achieve eruption/extrusion of the impacted canine (i.e. from surgical exposure to bonding a bracket on the middle of the labial surface) was $3.05 \pm 1.07$ for group 1, and 6.86 \pm 4.53 months for group 2 ( $\mathrm{P}<0.01$ ). Bone support did not differ significantly between the test groups, but in comparison with the contralateral side, differences were found at the mesial side of the canine and the distal side of the lateral incisor.

The prospective study by $\mathrm{AZ} \mathrm{Oz}$ et al [8] evaluated the periodontal status of impacted canines, the thickness of the adjacent buccal bone, and incidence of root resorption of the adjacent incisors in the 20 patients tested. Two sets of CBCT images were made for each patient. The first (T0) was taken before the start of orthodontic treatment, and the second (T1) after 3 weeks of retention. Alveolar bone loss (ABL) was measured on the buccal, palatal, mesial, and distal surfaces of previously impacted and contralateral canines on the CBCT images. All measurements were made twice, and the mean values were recorded. The evaluation of periodontal status revealed that the mean PD was deeper in the previously impacted canines $(2.13 \mathrm{~mm})$ compared with the contralateral canines $(1.64 \mathrm{~mm})$. A significant difference in ABL was observed on the mesial, distal, and palatal surfaces of previously impacted canines versus contralateral canines.

A-C. DA SILVA et al [9] found no statistically significant difference in 16 patients at a mean of 5 years and 11 months after restraint. They have performed measurements in tomographic scans to compare, in a long term-basis, root length and alveolar bone level in canines, lateral incisors, and first premolars (both sides). The highest alveolar losses found by these authors were 0.03 $\mathrm{mm}$ in the vestibular of the first premolars and $0.39 \mathrm{~mm}$ in the palatal of the lateral incisors. 
Only one out of four studies evaluated the bone outcomes of vestibular inclusion. J-Y Lee's retrospective study of 54 patients [10] found that the distance between the amelocemental junction and the alveolar crest was 0.82-
$0.89 \mathrm{~mm}$ longer and the root length $1.78 \mathrm{~mm}$ shorter in the inclusion group than in the non-inclusion group. In addition, the closed technique showed a slightly better result than the other techniques.
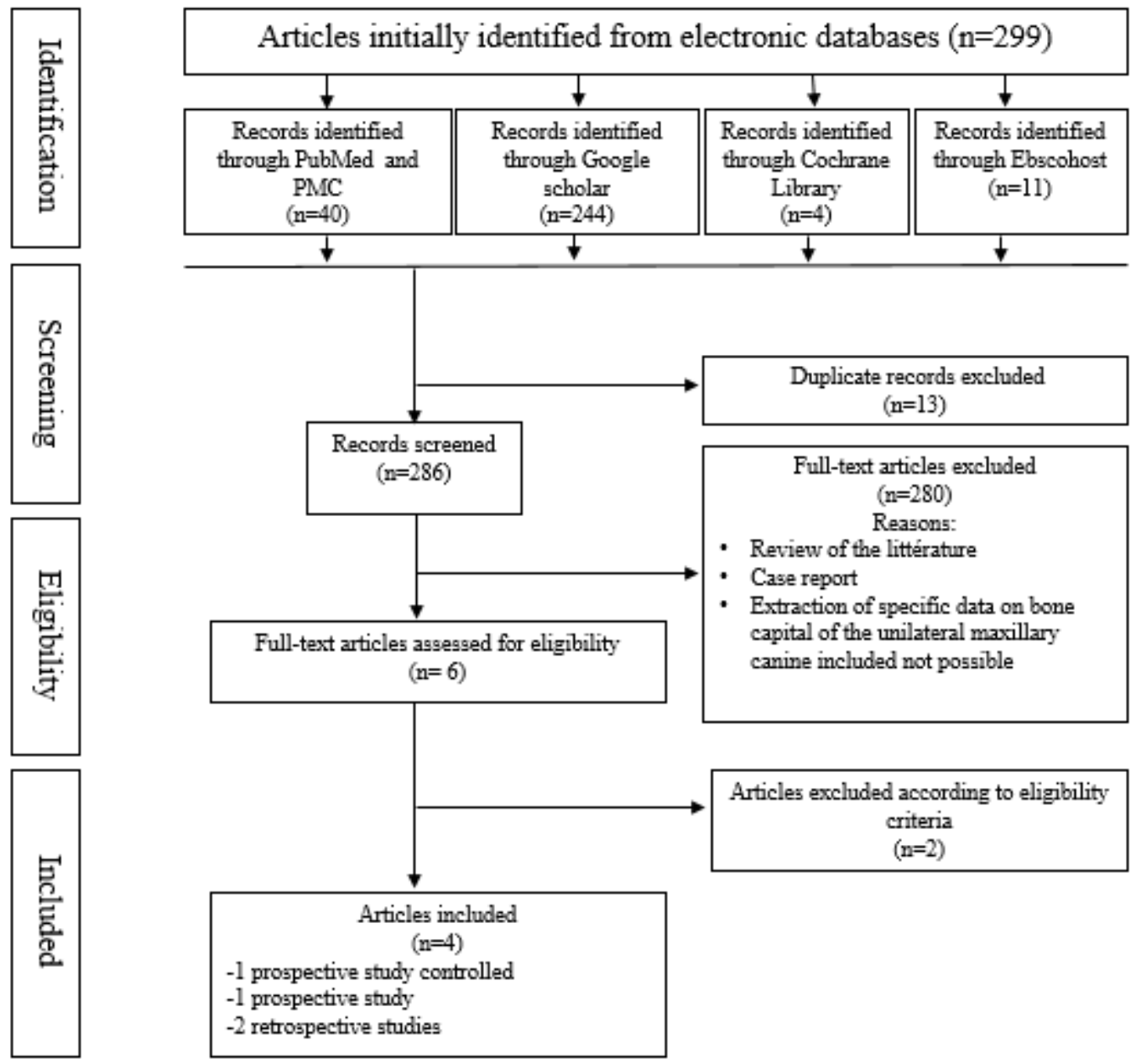

Figure 1: PRISMA flow diagram of the literature search and selection process.

\section{DISCUSSION}

Many studies were designed to compare the post-treatment periodontal status of palatally impacted canines treated by two different surgical methods: an open technique with the free eruption and closed flap technique. That is the case of D. Smailiene et al study, which found contradictory answers of the bone capital after traction of an inclusive canine on the palatal side. The radiographic findings confirmed the clinical results: while the loss of bone support did not differ with respect to the surgical method, there was less bone support in quadrants with impacted canines on the mesiopalatal side than in quadrants with normally erupted canines. Similar results were reported by A. Caprioglio [11] and Schmidt and Kokich [12] reported that compared with contralateral control teeth, loss of bone support on the impacted side was significantly greater at the distopalatal aspect of the lateral incisors and the distobuccal aspect of the premolars. The level of crestal bone on the distal aspect of the lateral incisor on the affected side was lower than on the control side in our study. Duration of treatment was greater in the closed surgery group, but the difference was not significant. The results of this study showed no association between the duration of treatment and the patient's age at the start of treatment, nor on the initial localization (vertical and horizontal) of the impacted tooth.

On the other hand, $\mathrm{AZ} \mathrm{Oz}$ et al [8] used pre-and post-treatment three-dimensional records to compare the buccal bone thickness and periodontal status of impacted canine teeth versus contralateral canines. In this clinical study, they compared the thickness of the buccal bone adjacent to impacted canines with that of the contralateral canines. Bone thickness was significantly different between the impacted and contralateral canines in the apical, distal, mesial, and palatal region. This finding may result from differences in torque between the treated and contralateral canines. The explanation for the palatal bone loss might be that the orthodontic forces applied in buccal and occlusal directions can tip the tooth root palatally, which can damage the periodontal tissues. As reported in a previous study [13], it is difficult to move the root of the treated canine buccally. Although additional torque was applied and delivered with rectangular stainless steel archwires, a loss of torque might have occurred. Another explanation might be that long-term periodontal health can be influenced by orthodontic mechanics; root torque movements applied to the impacted canine decrease the bone support more than tipping or extrusive movements [14-15]. 


\section{$\underline{\text { Table } 2}$}

\begin{tabular}{|c|c|c|c|c|c|}
\hline & 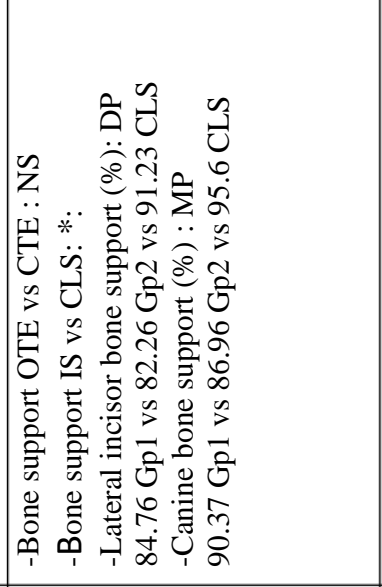 & 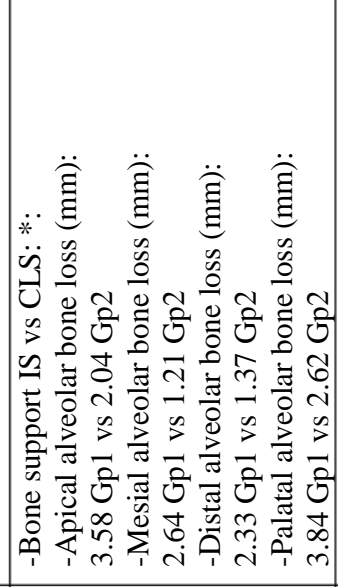 & 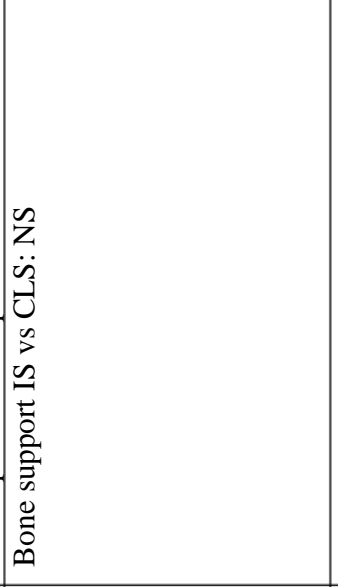 & 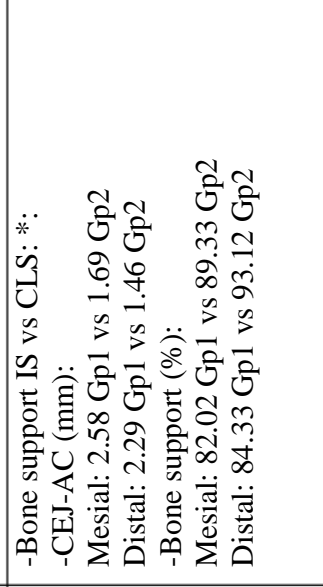 & 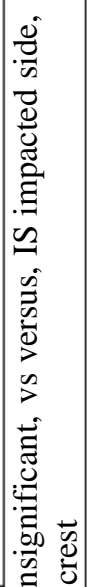 \\
\hline 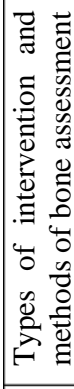 & 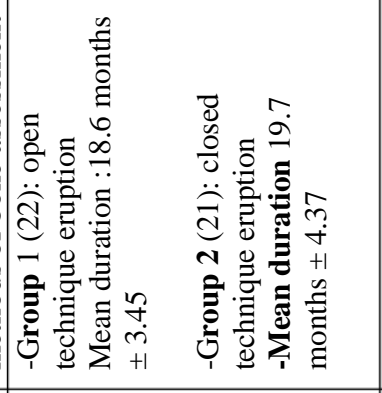 & 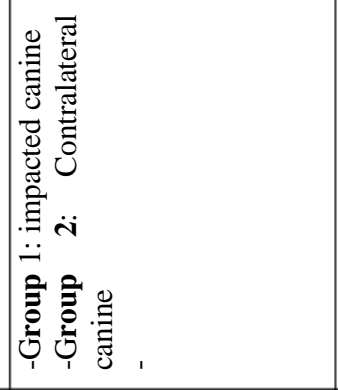 & 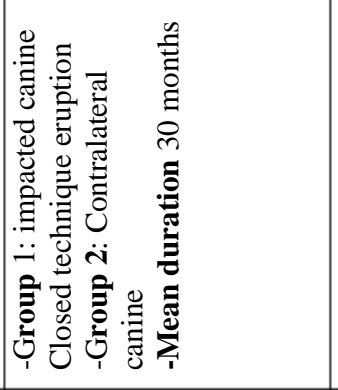 & 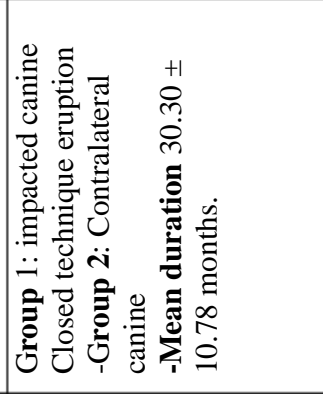 & 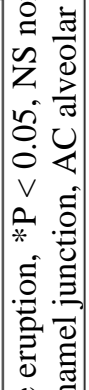 \\
\hline 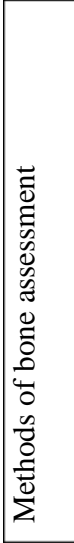 & 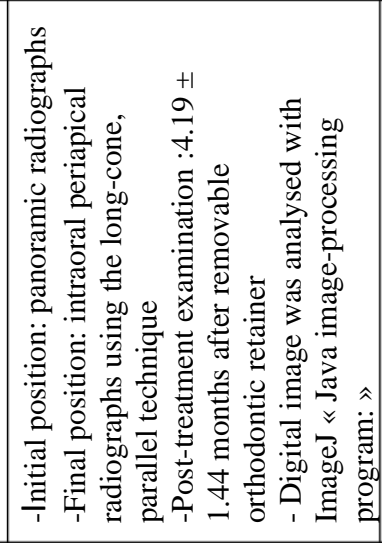 & 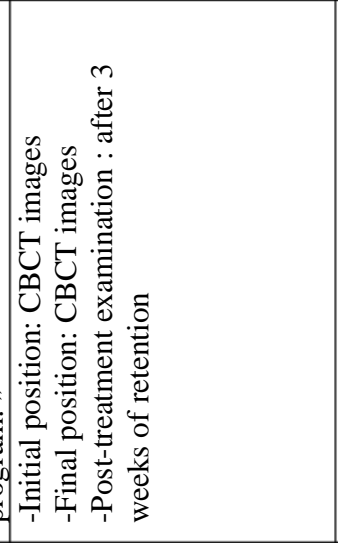 & 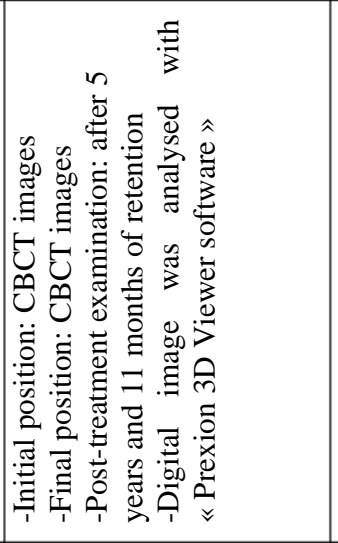 & 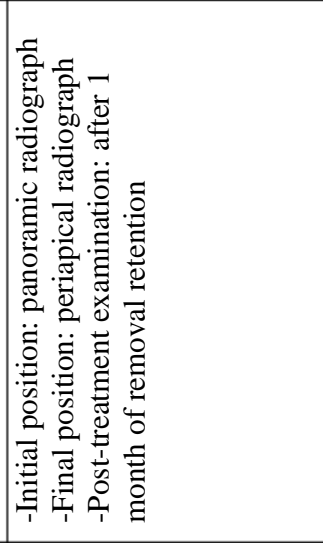 & 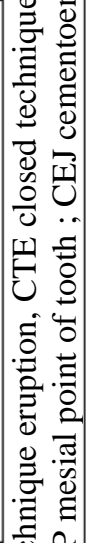 \\
\hline 吾 & 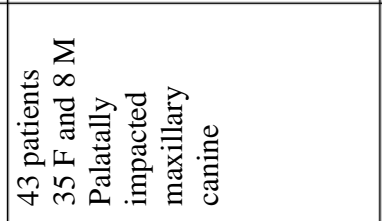 & 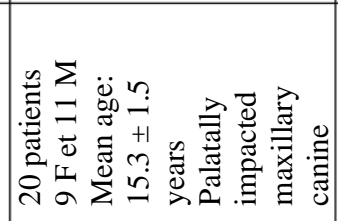 & 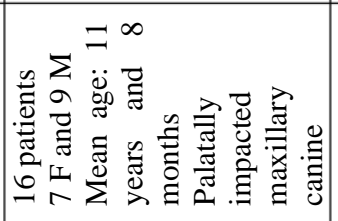 & 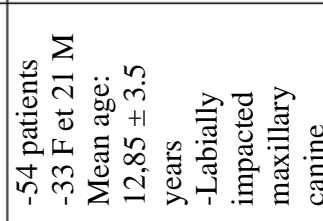 & 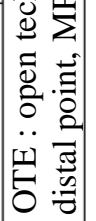 \\
\hline 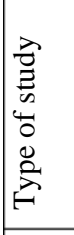 & 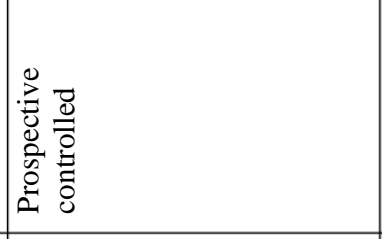 & 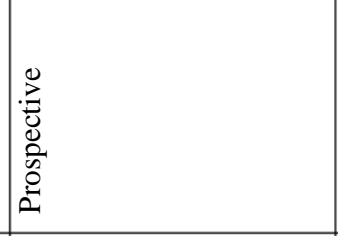 & 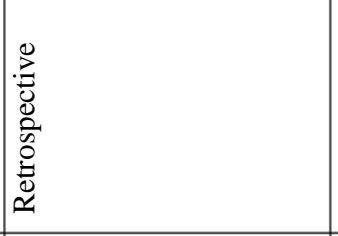 & 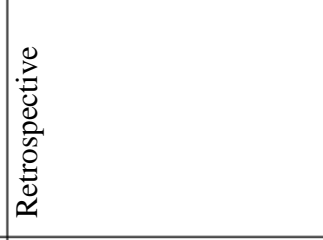 & 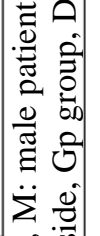 \\
\hline & 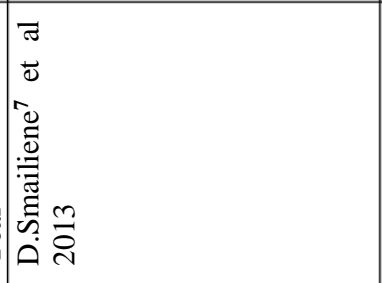 & 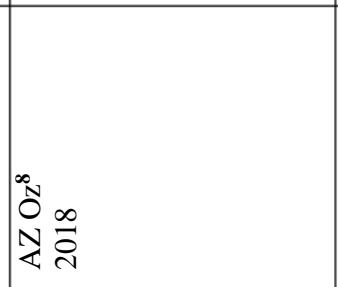 & 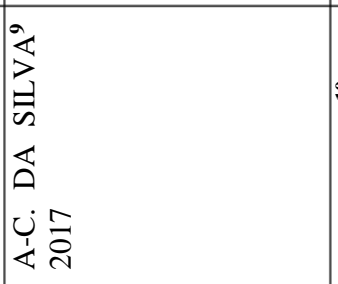 & 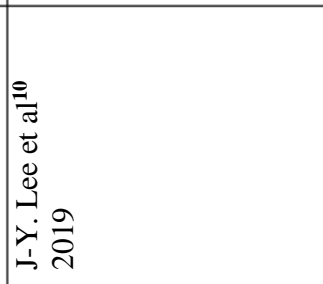 & 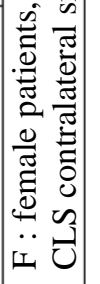 \\
\hline
\end{tabular}


However, A-C. DA SILVA [9] using the same treatment three-dimensional records than $\mathrm{AZ} \mathrm{Oz}$ et al [8] to compare the bone thickness and periodontal status of impacted canine teeth versus contralateral canines found a statically non-significant difference with the contralateral canine which is normally located on the arch on the bony support plane. In this study, a closed-eruption technique associated with a canine crown perforation performed is highlighted. A segmented arch and the use of a transpalatal arch as an anchorage device was the selected mechanics for the orthodontic traction of impacted canines. These results are similar to those reported by Schmidt and Kokich when evaluating the mesial and distal bone level using periapical radiographs [ 12]. The same results were found by Caprioglio, Vanni and Bolamperti [11] evaluating palatally impacted canines that suffered traction. On the other hand, Becker and Chaushi [ 16] and Evren, et al [ 17], assessing the mesial and distal bone level in canines that suffered traction, also in periapical radiographs, found a statistically significant bone loss among groups. On the latest, not only palatally, but buccally displaced canines presented reduced bone levels compared with their contralaterals. It should be highlighted that palatally displaced canines, when suffering traction, may not compromise the periodontal status as those buccally displaced. Some conflicting findings could be accounted by the method of evaluation, either CBCT scans or periapical radiograph, which could stand for such difference.

As for the results concerning vestibular inclusion, only one study in our review reported bony results [10]. J-Y. Lee et al [10] compared the periodontal results of a vestibular included canine tooth pulled by a closed technique with its

\section{REFERENCES}

[1] Alhammadi M-S, Asiri H-A et A-A.Almashraqi.Incidence, severity and orthodontic treatment difficulty index of impacted canines in Saudi population. J Clin Exp Dent. 2018;10(4):327-34.

[2] Manne R, Gandikota C-S, Anche S et al. Impacted canines: Etiology, diagnosis, and orthodontic management. J Pharma Bioallied Sci 2012; 4(2): 234-238.

[3] Kokich VG. Surgical and orthodontic management of impacted maxillary canines. Am J Orthod Dentofacial Orthop 2004;126: 278-83.

[4] Kokich V G. Preorthodontic uncovering and autonomous eruption of palatally impacted maxillary canines. Semin Orthod 2010; 16:205-211.

[5] Parkin N-A, Milner R-S, Deery C et al. Periodontal health of palatally displaced canines treated with open or closed surgical technique: A multicenter, randomized controlled trial. Am J Orthod Dentofacial Orthop 2013;144:176-84.

[6] Incerti-Parenti S, Checchi V, Ippolito D-R et al. Periodontal status after surgical-orthodontic treatment of labially impacted canines with different surgical techniques: A systematic review. Am J Orthod Dentofacial Orthop 2016;149:463-72.

[7] Smailiene D, Kavaliauskiene A, Pacauskiene I et al. Palatally impacted maxillary canines: choice of surgicalorthodontic treatment method does not influence post-treatment periodontal status. A controlled prospective study. European Journal of Orthodontics 2013;35:803810 .

[8] AZ Oz et Ciğer S. Health of Periodontal Tissues and Resorption Status after Orthodontic Treatment of Impacted Maxillary Canines. Niger J Clin Pract 2018;21:301-5

[9] DA SILVA A-C, CAPISTRANO A, De ALMEIDAPEDRIN R-R et al. Root length and alveolar bone level of impacted canines and adjacent teeth after orthodontic normally located contralateral. They concluded that the alveolar ridges are reduced when the canine is deeply embedded and that the distal alveolar ridge is likely to be resorbed the more the tooth is mesially inclined. Among the studies not included, S. Incerti Parenti [6] found that gingivectomy has more unfavorable periodontal outcomes compared to the apically displaced flap and the closed technique. This could be justified by the fact that in the vestibular, there is less attached gingiva than in the palatal area [6].

\section{CONCLUSION}

The results of our systematic review showed that there is a statistically significant difference in the bone between vestibular and palatal canine traction compared to contralateral traction normally placed on the dental arch. Canine traction is accompanied by statistically significant bone loss compared to contralateral canine traction. This bone defect is more aggravated when the inclusion is vestibular. However, this difference is less than $1 \mathrm{~mm}$ and therefore clinically acceptable. Further studies should be conducted to evaluate the long-term stability of orthodontically towed maxillary canines

\section{AUTHORS' CONTRIBUTIONS}

The participation of each author corresponds to the criteria of authorship and contributorship emphasized in the Recommendations for the Conduct, Reporting, Editing, and Publication of Scholarly work in Medical Journals of the International Committee of Medical Journal Editors.

\section{COMPETING INTERESTS}

The authors declare no competing interests with this case.

traction: a long-term evaluation.J Appl Oral Sci 2017; 25(1):75-81.

[10] Lee JY, Choi YJ, Choi SH et al. Labially impacted maxillary canines after the closed eruption technique and orthodontic traction: a split-mouth comparison of periodontal recession. J Periodontol 2019; 90(1):35-43.

[11] Caprioglio A, Vanni A, Bolamperti L. Long-term periodontal response to orthodontic treatment of palatally impacted maxillary canines. Eur J Orthod 2013; 35(3):3238.

[12] Schmidt A D, Kokich V G 2007 Periodontal response to early uncovering, autonomous eruption, and orthodontic alignment of palatally impacted maxillary canines. American Journal of Orthodontics and Dentofacial Orthopedics 131: e449-e455.

[13] Schmidt AD, Kokich VG. Periodontal response to early uncovering, autonomous eruption, and orthodontic alignment of palatally impacted maxillary canines. Am J Orthod Dentofacial Orthop 2007; 131:449-55. 14. Kohavi D, Becker A, Zilberman Y. Surgical exposure, orthodontic movement, and final tooth position as factors in periodontal breakdown of treated palatally impacted canines. Am J Orthod 1984;85:72-7.

[14] Steiner GG, Pearson JK, Ainamo J. Changes of the marginal periodontium as a result of labial tooth movement in monkeys. J Periodontol 1981;52:314-20.

[15] Becker A, Chaushu S. long-term follow-up of severely resorbed maxillary incisors after resolution of an etiologically associated impacted canine. Am J Orthod Dentofacial Orthop. 2005;127:650-4.

[16] Evren DA; Nevzatoglu S, Arun T, Acar A. periodontal status of ectopic canines after orthodontic treatment. Angle Orthod. 2014;84:18-23. 